July 17, 2012, v2346

\title{
Effective range corrections from effective field theory with di-baryon fields and perturbative pions
}

\author{
Shung-Ichi Ando ${ }^{1}$ and Chang Ho Hyun ${ }^{2}$ \\ Department of Physics Education, Daegu University, Gyeongsan 712-714, \\ Republic of Korea
}

\begin{abstract}
Contributions of perturbative pions around a non-trivial fixed point are studied by utilizing di-baryon fields. We calculate ${ }^{1} S_{0}$ and ${ }^{3} S_{1}$ phase shifts for the $n p$ scattering at low energies up to one-pion-exchange contributions. We also calculate effective range parameters, $v_{2}, v_{3}$, and $v_{4}$, in the higher order of the effective range expansion, and obtain corrections of the effective range to the expressions previously reported by Cohen and Hansen. After the scattering length and the effective range are renormalized, we study the role of renormalization scale parameter $\mu$ and we discuss the range of validity of the theory.
\end{abstract}

PACS(s): 11.10.Gh, 13.85.Dz, 21.45.Bc

\footnotetext{
${ }^{1}$ sando@daegu.ac.kr

${ }^{2}$ hch@daegu.ac.kr
} 


\section{Introduction}

The renormalization group analysis of two-nucleon interaction by Birse, McGovern, and Richardson [1] reveals that there are two fixed points, where no scales appear and a perturbative expansion is possible in the scale free limit. Around one fixed point, so called the trivial fixed point at which all the interactions vanish, a usual perturbation theory such as QED, chiral perturbation theory (ChPT) for meson sector and one nucleon sector $[2,3]$ can be constructed. Around the other fixed point, so called the non-trivial fixed point, the inverse of a scattering length or a binding energy vanishes for two-nucleon systems, and an effective range expansion (ERE) $[4,5]$ has been known for a long time in this limit.

After S. Weinberg had suggested an application of ChPT to nuclear physics, especially to nuclear forces two decades ago [6,7], a lot of works have been done so far. (For reviews, see, e.g., Refs. [8, 9, 10] and references therein. ${ }^{3}$ ) Because pions appear as (massless) Goldstone bosons due to spontaneous breaking of chiral symmetry of QCD (finite pion mass comes out due to explicit chiral symmetry breaking terms, i.e., quark masses), the pions are expected to play a dominant role in the long-range interactions. However, a difficulty of systematic application of ChPT to the two-nucleon system stems from the appearance of small scales such as large $S$-wave scattering length and small binding momentum of the deuteron, which are much smaller than the pion mass, $m_{\pi} \simeq 140 \mathrm{MeV}$. A fine-tuning is required in reproducing those small scales in the $S$-wave two-nucleon systems, which implies that the low energy constants can be important as well $[11,12]$.

In this work, we revisit the $S$-wave nucleon-nucleon scattering with perturbative pions proposed by Kaplan, Savage, and Wise (KSW) [13, 14]. We adopt the limit of the nontrivial fixed point as the "fine-tuned" ground state of the two nucleon system, utilize dibaryon fields $[15,16,17,18]$, which have the same quantum numbers of two-nucleon ${ }^{1} S_{0}$ and ${ }^{3} S_{1}$ states and the infinite scattering length or the zero binding energy, as a zeroth order two-nucleon core, and incorporate pion clouds perturbatively (one-pion-exchange contribution in this work as the first chiral correction ${ }^{4}$ ) around the non-trivial fixed point being represented by the di-baryon fields.

This may not be so unrealistic choice because it was conjectured by Braaten and Hammer that "Small increases in the up and down quark masses of QCD would tune the theory to the critical renormalization group trajectory for an infrared limit cycle in the three-nucleon system. At critical values of the quark masses, the deuteron binding energy goes to zero and the triton has infinitely many excited states with an accumulation point at the three-nucleon threshold" [19]. Subsequently, a study with an updated chiral effective theory reported an estimated critical pion mass, which makes binding energies of the deuteron and its isospin partner zero, tuned to be $m_{\pi}^{\text {crit }} \simeq 198 \mathrm{MeV}$ [20]. Thus the physical pion mass is closer to the critical pion mass than the mass in the chiral limit.

In addition, as shown in Ref. [21], because the expansion around the non-trivial fixed point is equivalent to ERE and takes a form of series of the inverse of potential (or the

\footnotetext{
${ }^{3}$ There have been intensive discussions concerning renormalization and counting rules, with a finite or infinite cutoff, in solving the Lippmann-Schwinger equation with nucleon-nucleon interactions obtained in chiral effective theory. For more details, see a recent review, e.g., Ref. [10], and references therein.

${ }^{4}$ See footnote 6 .
} 
on-shell $\mathrm{K}$ matrix), $1 / \mathrm{V}$, when one renormalizes coefficients of the ordinary contact interactions by using the effective range parameters, one has complicated nonlinear relations of the coefficients. On the other hand, because our expansion scheme matches well to ERE we obtain a linear relation among the coefficients if we include the di-baryon fields in the formalism up to the order we considered in this work. Thus the formalism with di-baryon fields provides us a way simple and transparent to account for the role of onepion exchange perturbatively in studying the chiral corrections in nuclear systems at low energies.

We calculate the $S$-wave phase shifts of the $N N$ scattering for ${ }^{1} S_{0}$ and ${ }^{3} S_{1}$ channels up to one-pion exchange contributions in the framework mentioned above, ${ }^{5}$ where $D$-wave mixture in the spin triplet channel is neglected for simplicity. ${ }^{6}$ We also calculate higher order terms of the effective range corrections, $v_{2}, v_{3}$, and $v_{4}$, whose definitions will be given below. Those terms have been calculated by Cohen and Hansen [24, 25] with a pionful effective field theory (EFT). In this work, we obtain new corrections to them from the effective ranges. After the scattering length and the effective range are renormalized by using empirical values, we retain the $\mu$-dependence in the effective range corrections, $v_{2}$, $v_{3}$, and $v_{4}$, and study the role of the scale parameter $\mu$ in the dimensional regularization (DR) and power divergence subtraction (PDS) scheme.

Usually, in a perturbative calculation, the same couplings appearing in higher orders as those being renormalized in lower orders by physical quantities, the scattering length and the effective range in the present case, are replaced by the renormalized couplings, and thus explicit $\mu$-dependence can be removed from an amplitude. However, the higher order contributions in the $S$-wave nucleon-nucleon scattering amplitude converge slowly and would bring a significant modification to the amplitude, thus, in this work we rather retain the $\mu$-dependence in the higher orders. In other words, the renormalized amplitude is perturbatively scale-independent, and adjusting the scale parameter $\mu$ can be regarded as a probe of "optimal higher order contributions". In general, terms corresponding to the scattering length, the effective range, and the higher order corrections, $v_{2}, v_{3}$, and $v_{4}$, in the calculation are functions of the scale parameter $\mu$. After renormalization of the scattering length and the effective range, we find that the higher order corrections, $v_{2}$, $v_{3}$ and $v_{4}$, are significantly sensitive to the value of $\mu$, and the sensitivity has a strong correlation to the range where the theory has predictive power.

This work is organized as the following. In section 2, we review pionless effective theory with di-baryon fields and briefly discuss relations between the formalism and the nontrivial fixed point. In section 3, we calculate the nucleon-nucleon scattering amplitudes

\footnotetext{
${ }^{5}$ We basically employ the standard counting rules suggested by KSW $[13,14]$. However, as to be mentioned, we do not calculate all of the contributions up to next-to-leading order (NLO) in the KSW counting rules, and expand the one-pion-exchange corrections around the inverse of the LO amplitude, $A_{d}^{-1}$. Thus two-pion-exchange contributions which would be important are counted as next-to-next-to leading order (NNLO) corrections.

${ }^{6}$ It is known that the one-pion exchange potential has a non-perturbative short range contribution, the strong attractive tensor force, in ${ }^{3} S_{1^{-}}{ }^{3} D_{1}$ channel [12], and the failure of the perturbative treatment of the one-pion exchange contribution in the higher order is reported in Ref. [22]. However, this difficulty can be avoided by introducing a regulator to the singular part of the one-pion exchange potential [23].
} 
for ${ }^{1} S_{0}$ and ${ }^{3} S_{1}$ channel with the one-pion-exchange contributions. In section 4 , the amplitudes are renormalized by using the empirical values of the scattering lengths and the effective ranges. In section 5 , the higher order effective range corrections, $v_{2}, v_{3}$, and $v_{4}$, are obtained, and the $\mu$ dependences of the effective range parameters and the phase shifts are studied. Finally, in section 6, discussion and conclusions are presented.

\section{Pionless theory with di-baryon fields}

In this section, we briefly review a pionless theory with di-baryon fields. A relevant effective Lagrangian reads $[16,17]$

$$
\mathcal{L}=\mathcal{L}_{N}+\mathcal{L}_{s}+\mathcal{L}_{t}
$$

where $\mathcal{L}_{N}$ is the Lagrangian for standard one nucleon sector, and $\mathcal{L}_{s}$ and $\mathcal{L}_{t}$ are those for the di-baryon fields in the ${ }^{1} S_{0}$ and the ${ }^{3} S_{1}$ states, respectively. The Lagrangian $\mathcal{L}_{N}$ in the heavy-baryon formalism reads

$$
\mathcal{L}_{N}=N^{\dagger}\left[i v \cdot \partial+\frac{(v \cdot \partial)^{2}-\partial^{2}}{2 m_{N}}\right] N,
$$

where $v^{\mu}$ is the velocity vector satisfying the condition $v^{2}=1$, and $m_{N}$ is the nucleon mass. The Lagrangians with the di-baryon fields read

$$
\begin{aligned}
& \mathcal{L}_{s}=\sigma_{s} s_{a}^{\dagger}\left[i v \cdot \partial+\frac{(v \cdot \partial)^{2}-\partial^{2}}{4 m_{N}}+\Delta_{s}\right] s_{a}-y_{s}\left[s_{a}^{\dagger}\left(N^{T} P_{a}^{\left({ }^{1} S_{0}\right)} N\right)+\text { h.c. }\right] \\
& \mathcal{L}_{t}=\sigma_{t} t_{i}^{\dagger}\left[i v \cdot \partial+\frac{(v \cdot \partial)^{2}-\partial^{2}}{4 m_{N}}+\Delta_{t}\right] t_{i}-y_{t}\left[t_{i}^{\dagger}\left(N^{T} P_{i}^{\left({ }^{3} S_{1}\right)} N\right)+\text { h.c. }\right]
\end{aligned}
$$

where $s_{a}$ and $t_{i}$ are the di-baryon fields for the ${ }^{1} S_{0}$ and ${ }^{3} S_{1}$ states, respectively, $\sigma_{s, t}=-1$, $\Delta_{s, t}$ are mass differences between the di-baryon masses and the two nucleon mass, and $y_{s, t}$ are coupling constants of di-baryon-nucleon-nucleon $(d N N)$ vertices. $P_{a}^{\left({ }^{1} S_{0}\right)}$ and $P_{i}^{\left({ }^{3} S_{1}\right)}$ are projection operators for ${ }^{1} S_{0}$ and ${ }^{3} S_{1}$ two-nucleon states, respectively, which read

$$
P_{a}^{\left({ }^{1} S_{0}\right)}=\frac{1}{\sqrt{8}} \tau_{2} \tau_{a} \sigma_{2}, \quad P_{i}^{\left({ }^{3} S_{1}\right)}=\frac{1}{\sqrt{8}} \tau_{2} \sigma_{2} \sigma_{i},
$$

where $\vec{\sigma}$ and $\vec{\tau}$ are Pauli matrices operating in the spin and isospin spaces, respectively.

Diagrams for dressed di-baryon propagator are shown in Fig. 1. ${ }^{7}$ As shown in Ref. [13], two-nucleon bubble diagrams are summed up to the infinite order, and thus the inverse of dressed di-baryon propagator in the center of mass frame is obtained as

$$
\begin{aligned}
i D_{s, t}^{-1}(p) & =i\left[\sigma_{s, t}\left(E+0+\Delta_{s, t}\right)+y_{s, t}^{2} \frac{m_{N}}{4 \pi}(\mu+i p)\right] \\
& =i y_{s, t}^{2} \frac{m_{N}}{4 \pi}\left[\frac{4 \pi \sigma_{s, t} \Delta_{s, t}}{m_{N} y_{s, t}^{2}}+\frac{4 \pi \sigma_{s, t}}{m_{N}^{2} y_{s, t}^{2}} p^{2}+\mu+i p\right] .
\end{aligned}
$$

Energy of the nucleon in the center of mass frame reads $E=p^{2} / m_{N}$, and we have employed DR and PDS scheme where $\mu$ is the renormalization scale for the loop integration. 


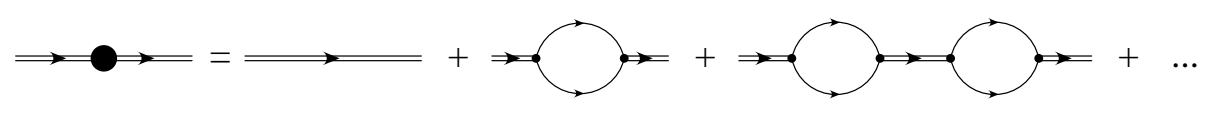

Figure 1: Diagrams for dressed di-baryon propagator: a double line denotes a di-baryon field and a line does a nucleon field. A double line with a filled circle denotes a dressed di-baryon field.

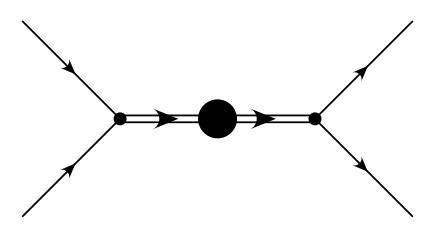

Figure 2: Diagram for S-wave NN scattering amplitude: a double line with a filled circle is the dressed di-baryon propagator obtained in Fig. 1.

A diagram for the $S$-wave $N N$ scattering is given in Fig. 2. Corresponding scattering amplitude is obtained as

$$
i A_{s, t}=\frac{4 \pi}{m_{N}} \frac{i}{-\mu-\frac{4 \pi \sigma_{s, t} \Delta_{s, t}}{m_{N} y^{2}}-\frac{4 \pi \sigma_{s, t}}{m_{N}^{2} y_{s, t}^{2}} p^{2}-i p} .
$$

On the other hand the $S$-wave amplitude in terms of the phase shift is given by

$$
A=\frac{4 \pi}{m_{N}} \frac{1}{p \cot \delta_{0}-i p},
$$

where $p \cot \delta_{0}$ is expanded in terms of the effective range parameters as

$$
p \cot \delta_{0}=-\frac{1}{a}+\frac{1}{2} r p^{2}+\cdots
$$

$a$ is the scattering length, $r$ is the effective range, and the dots denote higher order terms. With only di-baryon fields, Eq. (7) gives the corrections up to the quadratic term of $p$, i.e. $p^{2}$, but if we include the one-pion-exchange contributions, we have terms in higher orders of $p$. We note that the two nucleon bubble diagram plays a role similar to a kinetic term in mass renormalization in a propagator, and the scattering amplitude is normalized by the term proportional to -ip. Consequently, with the term proportional to $1 / y^{2}$ due to the normalization of the amplitude, we can easily make linear relations to the effective range terms being expanded in the denominator of the amplitude. Equating Eq. (7) with Eq. (8), we have

$$
-\frac{1}{a}=-\mu-\frac{4 \pi \sigma_{s, t} \Delta_{s, t}}{m_{N} y_{s, t}^{2}}, \quad r=-\frac{8 \pi \sigma_{s, t}}{m_{N}^{2} y_{s, t}^{2}} .
$$

\footnotetext{
${ }^{7}$ Diagrams are prepared by using a $\mathrm{C}++$ library, "FeynDiagram"[26].
} 


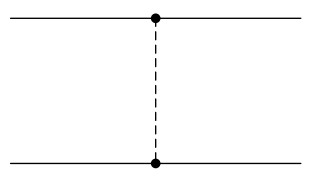

(I)

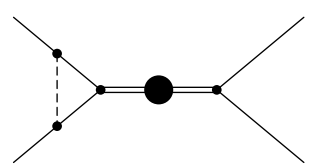

(II-a)

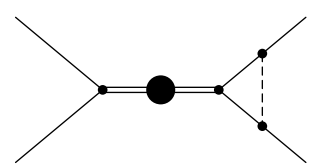

(II-b)

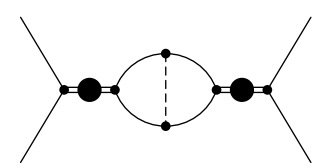

(III)

Figure 3: Diagrams for contributions from one-pion-exchange to the $N N$ scattering amplitude: a dashed line denotes a "potential" pion field. See the caption in Fig. 1 as well.

In general, Lagrangian for the di-baryons corresponding to the effective range parameters may be written $\mathrm{as}^{8}$

$$
\mathcal{L}_{d}=d_{a}^{\dagger} \sum_{n=0}^{\infty} D_{n}\left[i v \cdot \partial+\frac{(v \cdot \partial)^{2}-\partial^{2}}{4 m_{N}}\right]^{n} d_{a},
$$

where $d=s, t$. The terms of the Lagrangian only require the Galilean invariance for two nucleons, and $D_{n}$ are coefficients, with the factor $1 / y^{2}$ from the leading $d N N$ interaction, to match with the effective range parameters.

\section{Di-baryons and perturbative pions}

Effective Lagrangian with di-baryon fields and pions may read

$$
\mathcal{L}=\mathcal{L}_{\pi}+\mathcal{L}_{\pi N}+\mathcal{L}_{s}+\mathcal{L}_{t},
$$

where $\mathcal{L}_{\pi}$ and $\mathcal{L}_{\pi N}$ are the chiral Lagrangians for pion and one-nucleon sector, respectively. Because we do not consider pion loops, $\mathcal{L}_{s}$ and $\mathcal{L}_{t}$ takes the same form as given in Eqs. (3) and (4), respectively. ${ }^{9}$

Effective chiral Lagrangians $\mathcal{L}_{\pi}$ and $\mathcal{L}_{\pi N}$ are standard ones in the literature and we have

$$
\begin{aligned}
\mathcal{L}_{\pi} & =-f_{\pi}^{2} \operatorname{Tr}(\Delta \cdot \Delta)+\frac{f_{\pi}^{2}}{4} \operatorname{Tr}\left(\chi_{+}\right), \\
\mathcal{L}_{\pi N} & =N^{\dagger}\left[i v \cdot D-2 i g_{A} S \cdot \Delta\right] N+\frac{1}{2 m_{N}} N^{\dagger}\left[(v \cdot D)^{2}-D^{2}+\cdots\right] N,
\end{aligned}
$$

with $D_{\mu}=\partial_{\mu}+\Gamma_{\mu}$ where $\Gamma_{\mu}=\frac{1}{2}\left[\xi^{\dagger}, \partial_{\mu} \xi\right], \Delta_{\mu}=\frac{1}{2}\left\{\xi^{\dagger}, \partial_{\mu} \xi\right\}$, and $\chi_{+}=\xi^{\dagger} \chi \xi^{\dagger}+\xi \chi^{\dagger} \xi$; $\chi$ is the symmetry breaking term generating the pion mass, $\chi \propto m_{\pi}^{2}$ at leading order, and $\xi$ is the chiral field, $\xi \xi=\exp \left(i \vec{\tau} \cdot \vec{\pi} / f_{\pi}\right) . f_{\pi}$ is the pion decay constant and $g_{A}$ is the axial vector coupling constant. Dots denote terms irrelevant to this work.

With the one-pion-exchange contributions (so called "potential" pion) at $\mathcal{O}\left(Q^{0}\right)$ shown in Fig. 3, we may write down the whole amplitude as

$$
A=A_{d}+A_{\pi},
$$

\footnotetext{
${ }^{8}$ A slightly different expression of the Lagrangian for the deuteron pole has been obtained by Grießhammer. See Eq. (2.1) in Ref. [27].

${ }^{9}$ The chiral Lagrangians with di-baryon fields have been obtained by Soto and Tarrús [18, 28].
} 
where $A_{d}$ is the amplitude from the tree-level dressed di-baryon contributions without pions, $A_{s, t}$ in Eq. (7), and $A_{\pi}$ is the amplitude from the one-pion-exchange contributions in Fig. 3. We may divide it as

$$
A_{\pi}=A_{(I)}+A_{(I I)}+A_{(I I I)},
$$

where the amplitudes, $A_{(I)}, A_{(I I)}$, and $A_{(I I I)}$ are obtained as

$$
\begin{aligned}
A_{(I)=} & \left(\frac{g_{A}}{2 f_{\pi}}\right)^{2}\left[-1+\frac{m_{\pi}^{2}}{4 p^{2}} \ln \left(1+\frac{4 p^{2}}{m_{\pi}^{2}}\right)\right], \\
A_{(I I)=} & \frac{1}{O_{d}-i p}\left(\frac{g_{A}}{2 f_{\pi}}\right)^{2}\left\{-2 \mu+\frac{m_{\pi}^{2}}{p} \arctan \left(\frac{2 p}{m_{\pi}}\right)+2 i p\left[-1+\frac{m_{\pi}^{2}}{4 p^{2}} \ln \left(1+\frac{4 p^{2}}{m_{\pi}^{2}}\right)\right]\right\}, \\
A_{(I I I)=} & \frac{1}{\left(O_{d}-i p\right)^{2}}\left(\frac{g_{A}}{2 f_{\pi}}\right)^{2}\left\{-\mu^{2}+m_{\pi}^{2}+m_{\pi}^{2} \ln \left(\frac{\mu}{m_{\pi}}\right)-p^{2}\right. \\
& \left.+2 i p\left[-\mu+\frac{m_{\pi}^{2}}{2 p} \arctan \left(\frac{2 p}{m_{\pi}}\right)\right]-2 p^{2}\left[-1+\frac{m_{\pi}^{2}}{4 p^{2}} \ln \left(1+\frac{4 p^{2}}{m_{\pi}^{2}}\right)\right]\right\},
\end{aligned}
$$

with

$$
O_{d}=-\mu-\frac{4 \pi \sigma \Delta}{m_{N} y^{2}}-\frac{4 \pi \sigma}{m_{N}^{2} y^{2}} p^{2}
$$

The subscripts $s, t$ are dropped from the di-baryon parameters, $\sigma, \Delta$, and $y$ for simplicity. The loop diagrams have been calculated by using DR and PDS scheme, as done in Refs. [13, 14].

Expanding the one-pion-exchange amplitude $A_{\pi}$ around the inverse of the di-baryon amplitude $1 / A_{d}$, which is associated with the non-trivial fixed point, we have

$$
p \cot \delta_{0}=i p+\frac{4 \pi}{m_{N}} \frac{1}{A}=i p+\frac{4 \pi}{m_{N}} \frac{1}{A_{d}+A_{\pi}} \simeq i p+\frac{4 \pi}{m_{N}} \frac{1}{A_{d}}-\frac{4 \pi}{m_{N}} \frac{A_{\pi}}{A_{d}^{2}} .
$$

We note that because the one-pion-exchange correction is obtained in the form of $A_{\pi} / A_{d}^{2}$ in the above expression, the pole structures due to the di-baryon propagator in $A_{(I I)}$ and $A_{(I I I)}$ disappear, whereas $A_{d}^{-2}$ and $A_{d}^{-1}$ terms appear as interactions $\left(O_{d}^{2}\right.$ and $\left.O_{d}\right)$ along with $A_{(I)}$ and $A_{(I I)}$ terms, respectively.

Inserting Eqs. (7) and (17-19) into Eq. (21), we obtain

$$
\begin{aligned}
& p \cot \delta_{0}=O_{d}-\frac{g_{A}^{2} m_{N}}{16 \pi f_{\pi}^{2}}\left\{-\mu^{2}+m_{\pi}^{2}+m_{\pi}^{2} \ln \left(\frac{\mu}{m_{\pi}}\right)-p^{2}\right. \\
& \left.\quad+2 O_{d}\left[-\mu+\frac{m_{\pi}^{2}}{2 p} \arctan \left(\frac{2 p}{m_{\pi}}\right)\right]+\left(O_{d}^{2}-p^{2}\right)\left[-1+\frac{m_{\pi}^{2}}{4 p^{2}} \ln \left(1+\frac{4 p^{2}}{m_{\pi}^{2}}\right)\right]\right\} .
\end{aligned}
$$

We note that the right hand side of Eq. (9) is real, and Eq. (22) satisfies the unitary condition for the phase shift $\delta_{0}$ : the phase shift $\delta_{0}$ should be real below the threshold 
of pion production. Satisfying this condition is not so trivial, as recently pointed out in Ref. [29]. For example, in the scheme proposed by Kaplan, Savage and Wise [13], one performs resummation of the leading contact interaction $C_{0}$, and the next-leading contact interaction $C_{2}$ is treated perturbatively in calculation of the amplitude. The unitarity would be satisfied order by order in the perturbative expansion. This treatment makes the phase shift $\delta_{0}$ complex in the higher order corrections, and thus one needs an additional expansion of $\tan \delta_{0}$ in terms of the phase shift $\delta_{0}$.

\section{Scattering length and effective range: renormalization}

We renormalize our results with two effective range parameters, scattering length and effective range in the expression,

$$
p \cot \delta_{0}=-\frac{1}{a}+\frac{1}{2} r p^{2}+F(p),
$$

where $F(p)$ is a function for effective range parameters in higher orders of $p$; we can approximate $F(p) \propto p^{4}$ when $p \ll 1$.

If we define $a_{d}(\mu)$ and $r_{d}(\mu)$ as

$$
\begin{gathered}
\frac{1}{a_{d}(\mu)} \equiv\left\{\frac{1}{a}-\frac{g_{A}^{2} m_{N}}{16 \pi f_{\pi}^{2}}\left[-\mu^{2}+m_{\pi}^{2}+m_{\pi}^{2} \ln \left(\frac{\mu}{m_{\pi}}\right)\right]\right\}\left[1-\frac{g_{A}^{2} m_{N}}{8 \pi f_{\pi}^{2}}\left(-\mu+m_{\pi}\right)\right]^{-1}, \\
\frac{1}{2} r_{d}(\mu) \equiv\left\{\frac{1}{2} r+\frac{g_{A}^{2} m_{N}}{16 \pi f_{\pi}^{2}}\left[-1+\frac{8}{3} \frac{1}{m_{\pi} a_{d}(\mu)}-\frac{2}{m_{\pi}^{2} a_{d}^{2}(\mu)}\right]\right\}\left[1-\frac{g_{A}^{2} m_{N}}{8 \pi f_{\pi}^{2}}\left(-\mu+m_{\pi}\right)\right]^{-1},
\end{gathered}
$$

then we obtain the higher order contributions as

$$
\begin{aligned}
F(p)= & -\frac{g_{A}^{2} m_{N}}{16 \pi f_{\pi}^{2}}\left\{\left[-\frac{8}{3} \frac{1}{m_{\pi} a_{d}(\mu)}+\frac{2}{m_{\pi}^{2} a_{d}^{2}(\mu)}\right] p^{2}\right. \\
& +2\left(-\frac{1}{a_{d}(\mu)}+\frac{1}{2} r_{d}(\mu) p^{2}\right)\left[-m_{\pi}+\frac{m_{\pi}^{2}}{2 p} \arctan \left(\frac{2 p}{m_{\pi}}\right)\right] \\
& \left.+\left[\left(-\frac{1}{a_{d}(\mu)}+\frac{1}{2} r_{d}(\mu) p^{2}\right)^{2}-p^{2}\right]\left[-1+\frac{m_{\pi}^{2}}{4 p^{2}} \ln \left(1+\frac{4 p^{2}}{m_{\pi}^{2}}\right)\right]\right\} .
\end{aligned}
$$

For the renormalization of the scattering length $a$ and the effective range $r$, we use the empirical values of the parameters as

$$
\frac{1}{a_{0}}=-8.30(\mathrm{MeV}), \quad r_{0}=2.73(\mathrm{fm}),
$$

for the ${ }^{1} S_{0}$ channel and

$$
\frac{1}{a_{1}}=36.4(\mathrm{MeV}), \quad r_{1}=1.76(\mathrm{fm}),
$$

for the ${ }^{3} S_{1}$ channel[30]. Conventionally, as discussed in the introduction, the terms, $1 / a_{d}(\mu)$ and $r_{d}(\mu)$, in the function $F(p)$ are replaced by the physical quantities, $1 / a$ and 

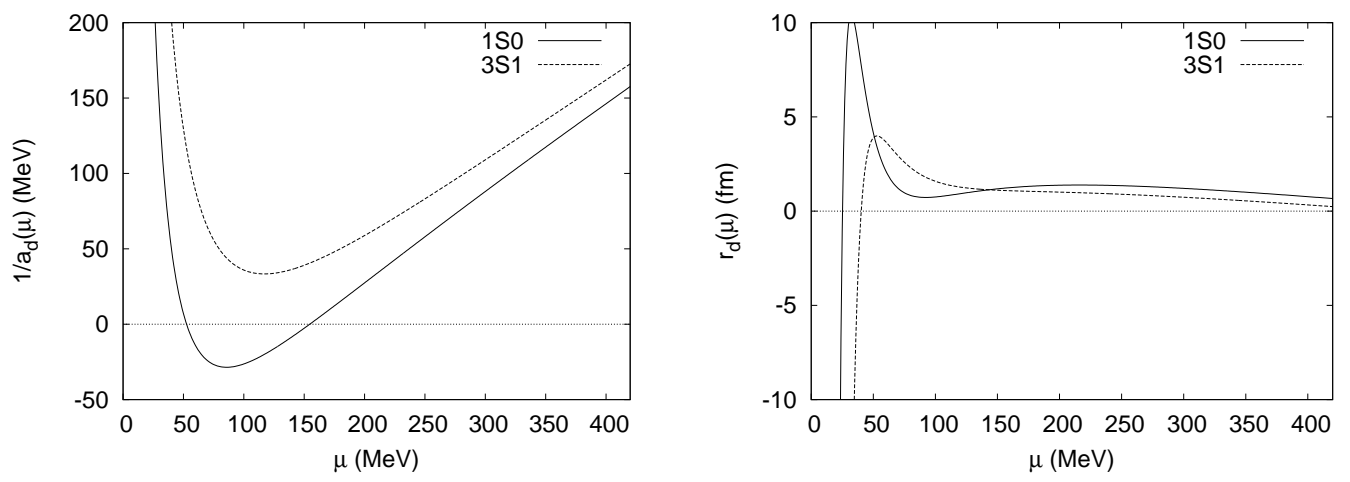

Figure 4: $1 / a_{d}(\mu)$ and $r_{d}(\mu)$ for ${ }^{1} S_{0}$ and ${ }^{3} S_{1}$ channels are plotted as functions of the scale parameter $\mu$.

$r$, respectively. In this work, we rather retain the $1 / a_{d}(\mu)$ and $r_{d}(\mu)$ terms in the higher orders as a probe of the optimal high energy and higher order contributions, and study the $\mu$-dependence in the higher order terms, the effective range corrections, $v_{2}, v_{3}$, and $v_{4}$, and the $\mathrm{S}$-wave phase shifts in higher momentum regions below.

The value of the scale parameter $\mu$ has been chosen as $\mu=m_{\pi}[13,14]$. With this particular choice of the scale parameter $\mu$, we have

$$
\frac{1}{a_{d}(\mu)}=\frac{1}{a_{0}} \text { and } \frac{1}{a_{1}}
$$

for the ${ }^{1} S_{0}$ and ${ }^{3} S_{1}$ channel, respectively. This implies that there are no contributions to $a_{d}(\mu)$ from the one-pion-exchange corrections, and it is determined solely by the dibaryon contribution. It was shown, e.g., in Ref. [31] that if the value of a scale parameter is chosen around the mass of the lightest degree of freedom that is integrated out, then the results for the phase shifts in the ${ }^{1} S_{0}$ channel agree very well with the experimental data. Because we consider only the one-pion-exchange in this work, we vary the scale parameter $\mu$ up to $\sim 400 \mathrm{MeV}$.

We plot $1 / a_{d}(\mu)$ and $r_{d}(\mu)$ as functions of $\mu$ in Fig. 4 . We can see that $1 / a_{d}(\mu)$ vanishes for the ${ }^{1} S_{0}$ channel at $\mu=52.6$ and $154.6 \mathrm{MeV}$, whereas there is no point where $1 / a_{d}(\mu)$ vanishes for the ${ }^{3} S_{1}$ channel.

For physically meaningful values of $\mu$, i.e. $\mu>m_{\pi}$, the value of $1 / a_{d}(\mu)$ increases monotonically as $\mu$ increases. At sufficiently large $\mu$, we can approximate Eq. (24) as

$$
\frac{1}{a_{d}(\mu)} \simeq \frac{1}{2} \mu
$$

and thus $1 / a_{d}(\mu)$ increases linearly with respect to $\mu$. For large $\mu$, we can approximate $r_{d}(\mu)$ as

$$
r_{d}(\mu) \simeq \frac{1}{2 \mu}\left(-1+\frac{4 \mu}{3 m_{\pi}}-\frac{\mu^{2}}{2 m_{\pi}^{2}}\right)
$$



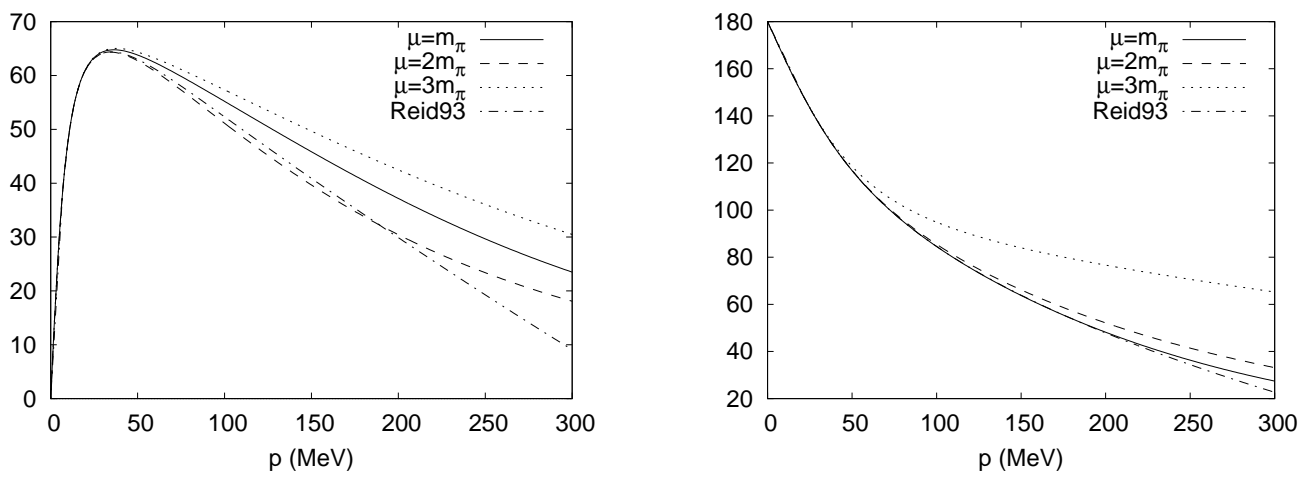

Figure 5: S-wave phase shifts for ${ }^{1} S_{0}$ channel (left panel) and for ${ }^{3} S_{1}$ channel (right panel): Curves of our results with $\mu=m_{\pi}, 2 m_{\pi}, 3 m_{\pi}$ are labeled by the $\mu$ values, and those labeled by "Reid93" are from a modern phenomenological potential.

Because $\mu$ 's in the parenthesis are divided by $m_{\pi}$ and we have $\mu^{-1}$ in front of the parenthesis, we have a relatively slow change of $r_{d}(\mu)$, and it eventually decreases because of the quadratic term in the parenthesis.

Fig. 5 shows the phase shifts in the ${ }^{1} S_{0}$ (left) and ${ }^{3} S_{1}$ (right) channels obtained from Eq. (23). If one requires that a result from EFT should be insensitive to the choice of a value of cutoff, corresponding to the scale parameter $\mu$ in this work, the applicable range of the present calculation for the $S$-wave phase shifts is quite restricted, less than $p \sim 50 \mathrm{MeV}$ when we choose the scale parameter $\mu$ as $\mu=m_{\pi}, 2 m_{\pi}$, and $3 m_{\pi}$, as shown in Fig. 5. However since we integrate out the degrees of freedom heavier than the one-pion exchange, it may be reasonable to consider the scale parameter in the range $m_{\pi}<\mu<2 m_{\pi}$. Within this range, ${ }^{1} S_{0}$ channel shows the best results with the $\mu$ close to $2 m_{\pi}$. This behavior is consistent with the one observed in Ref. [31]. For the ${ }^{3} S_{1}$ channel the results with reasonable range of $\mu$ show good agreement with each other to substantially large momenta.

It is worthwhile to consider the radius of convergence for which the perturbative expansion of Eq. (21) is reasonable. Since the scattering length and effective range terms are determined with the empirical values, the term $F(p)$ includes the contributions from one-pion exchange and depends on $\mu$. We consider the ratio defined by

$$
R \equiv \frac{|F(p)|}{-1 / a+r p^{2} / 2}
$$

as a criterion for the convergence.

Fig. 6 shows the ratio $R$ in the ${ }^{1} S_{0}$ channel with $\mu=m_{\pi}, 2 m_{\pi}$ and $3 m_{\pi}$. Dependence on the $\mu$ value is significant, but regardless of the $\mu$ value, one can say that the perturbative expansion of the pion-exchange contribution is reasonable for $p \leq 150 \mathrm{MeV}$. Agreement of the phase shifts to the phenomenological ones is good within this range. 


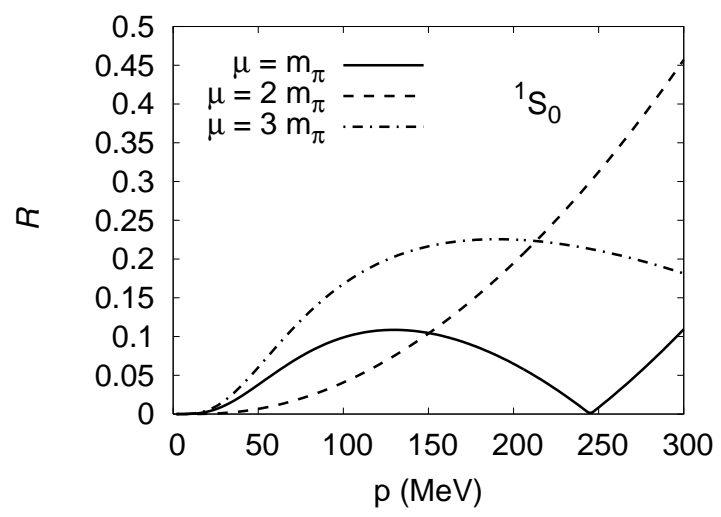

Figure 6: Ratio $R$ in Eq. (32) as a function of momentum $p$ with various values of $\mu$.

\section{Higher order effective range corrections}

After renormalization of the scattering length and the effective range, the function $F(p)$ in Eq. (26) has no unknown parameters, and depends only on the scale parameter $\mu$. Expanding $p \cot \delta_{0}$ to higher orders, one has

$$
p \cot \delta_{0}=-\frac{1}{a}+\frac{1}{2} r p^{2}+v_{2} p^{4}+v_{3} p^{6}+v_{4} p^{8}+\cdots .
$$

We renormalize the scattering length $a$ and the effective range $r$ to the physical values, and obtain the higher order parameters, $v_{2}, v_{3}$, and $v_{4}, \ldots$ by expanding $F(p)$ in Eq. (26) in powers of $p$ as

$$
F(p)=v_{2} p^{4}+v_{3} p^{6}+v_{4} p^{8}+\cdots
$$

We obtain

$$
\begin{aligned}
v_{2}= & \frac{g_{A}^{2} m_{N}}{16 \pi f_{\pi}^{2}}\left\{-\frac{16}{3} \frac{1}{a_{d}^{2}(\mu) m_{\pi}^{4}}+\frac{32}{5} \frac{1}{a_{d}(\mu) m_{\pi}^{3}}-\frac{2}{m_{\pi}^{2}}\left[1+\frac{r_{d}(\mu)}{a_{d}(\mu)}\right]+\frac{4}{3} \frac{r_{d}(\mu)}{m_{\pi}}\right\}, \\
v_{3}= & \frac{g_{A}^{2} m_{N}}{16 \pi f_{\pi}^{2}}\left\{16 \frac{1}{a_{d}^{2}(\mu) m_{\pi}^{6}}-\frac{128}{7} \frac{1}{a_{d}(\mu) m_{\pi}^{5}}+\frac{16}{3} \frac{1}{m_{\pi}^{4}}\left[1+\frac{r_{d}(\mu)}{a_{d}(\mu)}\right]\right. \\
& \left.-\frac{16}{5} \frac{r_{d}(\mu)}{m_{\pi}^{3}}+\frac{1}{2} \frac{r_{d}^{2}(\mu)}{m_{\pi}^{2}}\right\}, \\
v_{4}= & \frac{g_{A}^{2} m_{N}}{16 \pi f_{\pi}^{2}}\left\{-\frac{256}{5} \frac{1}{a_{d}^{2}(\mu) m_{\pi}^{8}}+\frac{512}{9} \frac{1}{a_{d}(\mu) m_{\pi}^{7}}-16 \frac{1}{m_{\pi}^{6}}\left[1+\frac{r_{d}(\mu)}{a_{d}(\mu)}\right]\right. \\
& \left.+\frac{64}{7} \frac{r_{d}(\mu)}{m_{\pi}^{5}}-\frac{4}{3} \frac{r_{d}^{2}(\mu)}{m_{\pi}^{4}}\right\} .
\end{aligned}
$$

We note that the expressions of $v_{2}, v_{3}$ and $v_{4}$ above are obtained in terms of $1 / a_{d}(\mu)$ and $r_{d}(\mu)$. Those expressions have been obtained by Cohen and Hassen without $r_{d}(\mu)$ and 
in terms of the physical scattering length $a[24,25]$. We can reproduce their results by choosing $\mu=m_{\pi}$ and neglecting the contribution $r_{d}(\mu)$.

Values of $v_{2}, v_{3}$, and $v_{4}$ are shown in Table 1 . The values in the first row for each channel are obtained from partial wave analysis (PWA). ${ }^{10}$ Those in the second row are obtained by putting $a_{d}(\mu)=a^{\text {phy. }}$ and $r_{d}(\mu)=0$ where $a^{\text {phy. }}$ is the physical value of the scattering length, $a^{p h y}$. $=a_{0}$ for the ${ }^{1} S_{0}$ channel and $a^{\text {phy }}=a_{1}$ for the ${ }^{3} S_{1}$ channel. These results are equal to what Cohen and Hansen have obtained [25]. Those in the third row are obtained by putting $a_{d}(\mu)=a^{p h y}$ and $r_{d}(\mu)=r^{p h y}$. where $r^{p h y}$. is the physical effective range, $r^{p h y}=r_{0}$ for the ${ }^{1} S_{0}$ channel and $r^{p h y}=r_{1}$ for the ${ }^{3} S_{1}$ channel. We find that the corrections from the effective range are significant, but it is not enough to reproduce the PWA values of $v_{2}, v_{3}$ and $v_{4}$. Those from the fourth to the eighth row are our results with various $\mu$ values, $\mu=m_{\pi}, 2 m_{\pi}, 3 m_{\pi}$, whereas $\mu=178$ and $330 \mathrm{MeV}$ for ${ }^{1} S_{0}$ channel and $\mu=167$ and $246 \mathrm{MeV}$ for ${ }^{3} S_{1}$ channel are fitted so that the values of $v_{2}$ of PWA are reproduced (we find several points to reproduce the value of $v_{2}$ of PWA as to be seen below). It has been recently discussed that the scale parameter may be considered as an additional short-range "counter term" provided one allows for a fine tuning of the scale value [32]. The results with $\mu=m_{\pi}, 2 m_{\pi}$ and $3 m_{\pi}$ show a sensitivity of $v_{2}, v_{3}$ and $v_{4}$ to the $\mu$ value, whereas the results fitted to the $v_{2}$ value of PWA fairly reproduce all of the values of $v_{2}, v_{3}$ and $v_{4}$ with the adjusted values of the scale parameter $\mu$.

Values of $v_{2}, v_{3}$, and $v_{4}$ in the ninth and tenth rows for the ${ }^{1} S_{0}$ channel in Table 1 are obtained by Fleming, Mehen, and Stewart (FMS) [22] up to next-to leading order (NLO) and next-to-next-to leading order (NNLO) in the KSW counting scheme, respectively. They employed so called global fitting method where they used the phase shift data in the ranges, $p=7 \sim 80 \mathrm{MeV}$ for $\mathrm{NLO}$ and $p=7 \sim 200 \mathrm{MeV}$ for NNLO, in fitting the parameters. In addition, they obtained slightly different effective ranges, $r=2.65 \mathrm{fm}$ for NLO and $r=2.63 \mathrm{fm}$ for NNLO from the empirical value $r=2.73 \mathrm{fm}$. Their NLO results basically agree with those obtained by Cohen and Hansen [24, 25], whereas the NNLO results are improved but not adequate to completely reproduce the results of PWA. It may indicate the slow convergence of the higher order corrections as discussed by the authors [22]. It is also noted that adjusting the $\mu$-value which improves the estimated values of $v_{2}, v_{3}$, and $v_{4}$ in this work seems to play a similar role to the higher order corrections.

Now we plot our results of $v_{2}, v_{3}$, and $v_{4}$ for ${ }^{1} S_{0}$ and ${ }^{3} S_{1}$ channels as functions of the scale parameter $\mu$ in Fig. 7 . We find the values of $v_{2}, v_{3}$, and $v_{4}$ are quite sensitive to the value of $\mu$ : the sensitivity increases as the order of the terms increases with the units we have chosen, $\mathrm{fm}^{3}, \mathrm{fm}^{5}, \mathrm{fm}^{7}$, respectively. Thus $v_{4}$, is the most sensitive, $v_{3}$ is in middle, and $v_{2}$ is less sensitive to $\mu$ than others.

The phase shifts for the $S$-waves are plotted in Fig. 8 with Eq. (23) and the $\mu$ adjusted to PWA $v_{2}$ values. Our results (labeled by "This work") are plotted with $\mu=178 \mathrm{MeV}$ for the ${ }^{1} S_{0}$ channel and $\mu=167 \mathrm{MeV}$ for the ${ }^{3} S_{1}$ channel. We compare our results with the ones which are obtained by keeping only two effective range parameters, $a$ and $r$ in

\footnotetext{
${ }^{10}$ The values of PWA are taken from Table I in Ref. [25].
} 


\begin{tabular}{|c|c|c|c|c|}
\hline & & $v_{2}\left(\mathrm{fm}^{3}\right)$ & $v_{3}\left(\mathrm{fm}^{5}\right)$ & $v_{4}\left(\mathrm{fm}^{7}\right)$ \\
\hline \multirow{10}{*}{${ }^{1} S_{0}$} & PWA & -0.48 & 3.8 & -17.0 \\
\hline & $a^{p h y}, r=0$ & -3.32 & 17.8 & -106.8 \\
\hline & $a^{p h y}, r^{p h y .}$ & 0.69 & 3.99 & -25.9 \\
\hline & $\mu=m_{\pi}$ & -1.74 & 11.1 & -68.0 \\
\hline & $\mu=2 m_{\pi}$ & 0.17 & 0.71 & -4.85 \\
\hline & $\mu=3 m_{\pi}$ & -2.75 & 17.8 & -117 \\
\hline & $\mu=178 \mathrm{MeV}$ & $-0.48^{*}$ & 4.40 & -26.8 \\
\hline & $\mu=330 \mathrm{MeV}$ & $-0.48^{*}$ & 4.37 & -29.0 \\
\hline & $\mathrm{FMS}(\mathrm{NLO})[22]$ & -3.3 & 19 & -117 \\
\hline & FMS(NNLO)[22] & -1.2 & 2.9 & -0.7 \\
\hline \multirow{8}{*}{${ }^{3} S_{1}$} & PWA & 0.04 & 0.67 & -4.0 \\
\hline & $a^{p h y}, r=0$ & -0.96 & 4.57 & -25.5 \\
\hline & $a^{p h y}, r^{p h y .}$ & 0.44 & 0.48 & -2.88 \\
\hline & $\mu=m_{\pi}$ & -0.05 & 1.42 & -8.22 \\
\hline & $\mu=2 m_{\pi}$ & -0.25 & 2.39 & -16.2 \\
\hline & $\mu=3 m_{\pi}$ & -3.23 & 21.2 & -139 \\
\hline & $\mu=167 \mathrm{MeV}$ & $0.04^{*}$ & 0.75 & -4.1 \\
\hline & $\mu=246 \mathrm{MeV}$ & $0.04^{*}$ & 0.68 & -4.7 \\
\hline
\end{tabular}

Table 1: Values of $v_{2}, v_{3}$, and $v_{4}$ for ${ }^{1} S_{0}$ and ${ }^{3} S_{1}$ channel: values in the first row for each of the channels are from partial wave analysis (PWA), those in the second row are obtained by putting $a_{d}(\mu)=a^{\text {phys. }}$ and $r_{d}(\mu)=0$ in our results, those in the third row by putting $a_{d}(\mu)=a^{\text {phys. }}$ and $r_{d}(\mu)=r^{\text {phys. }}$, and those from the fourth to the eighth row are our results with different $\mu$ values: for the fourth to the sixth row $\mu=m_{\pi}, 2 m_{\pi}$, and $3 m_{\pi}$, respectively, and those in the seventh and eighth row, $\mu$ value is fixed by using the value of $v_{2}$ of PWA (the values with * above are fitted ones); $\mu=178$ and $330 \mathrm{MeV}$ for ${ }^{1} S_{0}$ channel and $\mu=167$ and $246 \mathrm{MeV}$ for ${ }^{3} S_{1}$ channel. Values in the ninth and tenth rows for ${ }^{1} S_{0}$ channel are results obtained by Fleming, Mehen, Stewart (FMS) [22] up to NLO and $N N L O$ in the $K S W$ counting, respectively. 

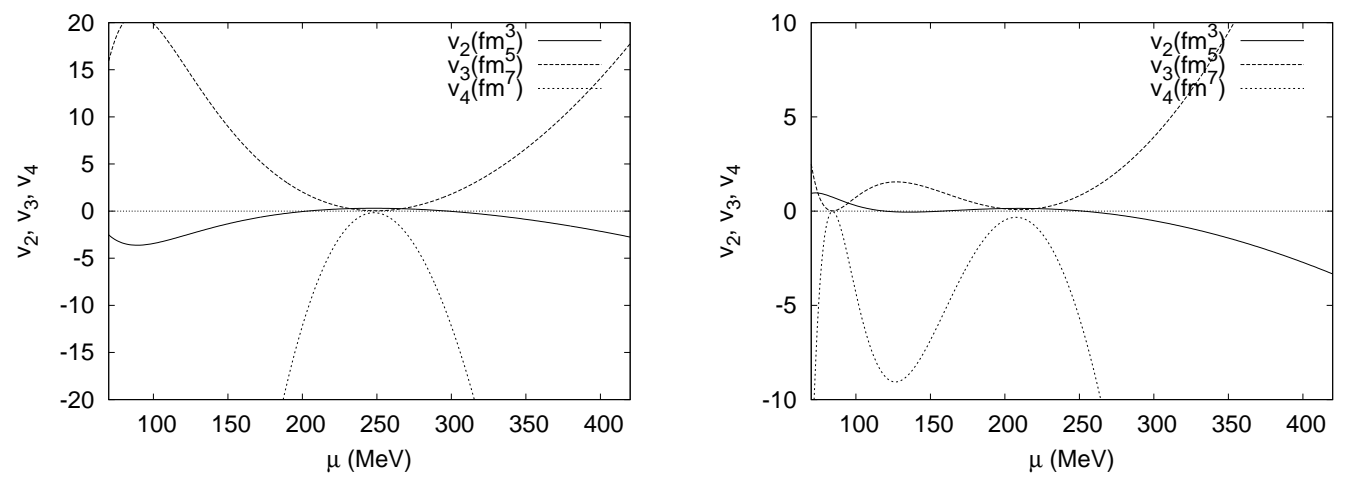

Figure 7: Effective range expansion parameters $v_{2}, v_{3}$, and $v_{4}$ for ${ }^{1} S_{0}$ channel (left panel) and ${ }^{3} S_{1}$ channel (right panel) are plotted as functions of the scale parameter $\mu$.

the effective range (ER) expansion, and those from a modern phenomenological potential (Reid93) [30]. Compared to the curve of ER, the results with one-pion exchange are improved in both accuracy and the range where the theory is applicable. Comparing the results with the fine-tuned $\mu$ values to those with randomly chosen $\mu$ 's in Fig. 5, we again see that both range and accuracy are improved. It is notable that the fine-tuned values of $\mu$ are within the range $m_{\pi}<\mu<2 m_{\pi}$, which may support the perturbative treatment of pion-exchange interactions in the framework of di-baryon pionless EFT.

Finally we investigate the contributions of $v_{2}, v_{3}$, and $v_{4}$ terms to the phase shifts. In Fig. 9 we plot the phase shifts in the ${ }^{1} S_{0}$ channel for ER, and $v_{2}, v_{3}$ and $v_{4}$ terms added successively with $\mu=178 \mathrm{MeV}$. The results are compared with those of Reid93. For the momenta $0<p<30 \mathrm{MeV}$, the results of $v_{2}, v_{3}$ and $v_{4}$ almost coincide. At around $p \sim 50$ $\mathrm{MeV}$, the variation from $v_{2}$ to $v_{3}$ is about half of that from ER to $v_{2}$, and the variation from $v_{3}$ to $v_{4}$ is much smaller than the previous ones. At $p=60 \mathrm{MeV}$, the variation from ER to $v_{2}$ is similar to that from $v_{2}$ to $v_{3}$, and the variation from $v_{3}$ to $v_{4}$ is almost half of $v_{2}$ and $v_{3}$. From around $p \sim 70 \mathrm{MeV}$, the variation from $v_{2}$ to $v_{3}$ exceeds that from ER to $v_{2}$, and from around $p \sim 80 \mathrm{MeV}$ the variation from $v_{3}$ to $v_{4}$ exceeds that from $v_{2}$ to $v_{3}$. Therefore we can say that the effective range expansion up to $v_{4}$ converges reasonably up to $p \sim 50 \mathrm{MeV}$, as previously pointed out in Ref. [33].

\section{Discussion and conclusions}

In this work, we calculate the phase shifts of the neutron-proton scattering for the ${ }^{1} S_{0}$ and ${ }^{3} S_{1}$ channels at low energies employing the di-baryon effective theory with perturbative pions. We include the one-pion-exchange diagrams, and expand their contributions around the inverse of the tree-level amplitude from the di-baryon fields to implement the expansion of the perturbative pions around the non-trivial fixed point. Loop diagrams are calculated by using DR and the PDS scheme, and we study our results by changing the value of the renormalization scale parameter $\mu$.

We renormalize the scattering length $a$ and the effective range $r$ to the physical values. 

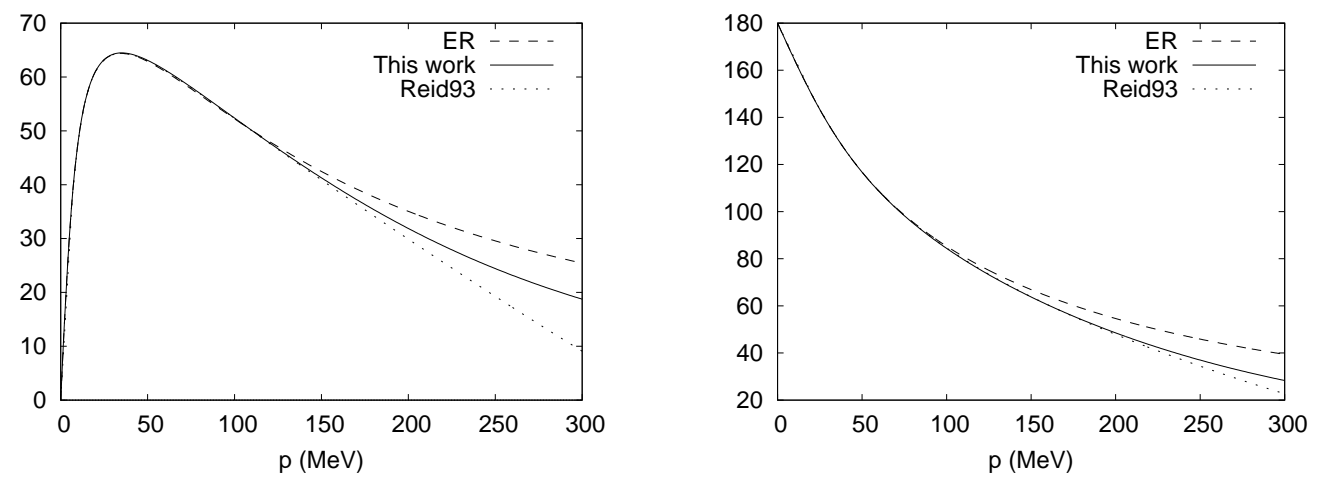

Figure 8: S-wave phase shifts for ${ }^{1} S_{0}$ channel (left panel) and for ${ }^{3} S_{1}$ channel (right panel): Curves labeled by "ER" are obtained from effective range expansion with the first two coefficients, scattering length and effective range, those by "This work" are our results using $\mu=178 \mathrm{MeV}$ for ${ }^{1} S_{0}$ and $\mu=167 \mathrm{MeV}$ for ${ }^{3} S_{1}$ channel, and those by "Reid93" are from a modern phenomenological potential.
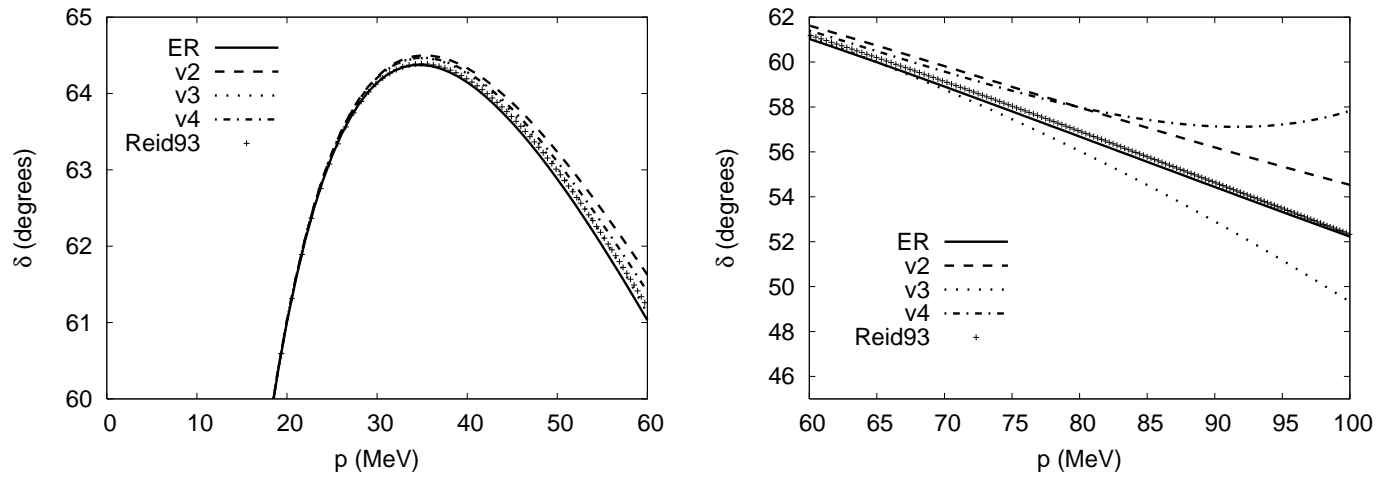

Figure 9: Phase shifts in the ${ }^{1} S_{0}$ channel for $E R, E R+v_{2}, E R+v_{2}+v_{3}$ and $E R+v_{2}+v_{3}+v_{4}$. Left panel shows the results for $0<p<60 \mathrm{MeV}$ and the right for $60<p<100 \mathrm{MeV}$. 
The scale dependence associated with the one-pion-exchange contributions are encoded in the functions $a_{d}(\mu)$ and $r_{d}(\mu)$ which we retain in the higher order terms of the effective range expansion as the probe of the optimal higher order corrections. These functions take part in the physical observable $p \cot \delta_{0}$ through the term denoted by $F(p)$. We also calculate the coefficients in the higher-order effective range corrections, $v_{2}, v_{3}$, and $v_{4}$. We obtain new corrections from $r_{d}(\mu)$ to the terms obtained by Cohen and Hansen [24, 25]. Those terms do not have unknown constants and are solely determined by the terms induced by the one-pion-exchange contributions. The $\mu$ dependences of $v_{2}, v_{3}$, and $v_{4}$ are plotted and we found the sensitivity to $\mu$. More sensitive dependence on $\mu$ is observed in the higher-order term with the units $\left(\mathrm{fm}^{3}, \mathrm{fm}^{5}, \mathrm{fm}^{7}\right) ; v_{4}$ is more sensitive than others, $v_{3}$ is medium, and $v_{2}$ is less sensitive and exhibits a mild $\mu$ dependence. By adjusting the $\mu$ value to the known $v_{2}$ value, however, we find that we can reasonably reproduce the $v_{3}$ and $v_{4}$ values obtained from partial wave analysis. If $\mu$ value is chosen randomly, it is hard to say that all the $v_{2}, v_{3}$ and $v_{4}$ values are well controlled and determined by the terms up to the one-pion-exchange contributions. In addition, this feature can be observed as well from the $\mu$ dependence of the phase shifts; applicable range of the present formalism up to the one-pion-exchange corrections is narrow, less than $p \sim 50 \mathrm{MeV}$ with $\mu=m_{\pi}, 2 m_{\pi}$ and $3 m_{\pi}$. However, if we use our best values of $\mu$ for $v_{2}$, then the range of the applicability of the theory is considerably widened, up to $p \sim m_{\pi}$.

Now we briefly discuss the work reported by Soto and Tarrus employing the di-baryon fields and the perturbative pions up to NNLO [28]. In that work, the authors included one higher order corrections than the present work, whereas they employed a slightly different counting rule, especially in the leading amplitude, $A_{d}$ in Eq. (7), and the different parameter fitting method. Because they focus on the momentum region, $p \sim m_{\pi}$, contributions from the effective range parameters, $1 / a$ and $r$, in $A_{d}$ are regarded as small corrections compared to the ip term generated from the nucleon bubble diagram, and are expanded as perturbative terms. Thus scattering amplitudes obtained in this expansion scheme do not reproduce the pole structures at very low energies in the S-wave nucleon-nucleon scattering, and they have fitted parameters by using the phase shifts data at $p=53 \sim$ $216 \mathrm{MeV}(E=3 \sim 50 \mathrm{MeV})$. In the NNLO calculation, they found similar results to those obtained in the KSW scheme without the di-baryon fields up to NNLO [22]; the phase shifts for the ${ }^{1} S_{0}$ channel are reproduced well up to $p \simeq 216 \mathrm{MeV}$ ( $\left.E \simeq 50 \mathrm{MeV}\right)$, whereas the calculated phase shifts for the ${ }^{3} S_{1}$ channel deviate from those obtained in the accurate potential models at higher momenta and are good up to $p \simeq 137 \mathrm{MeV}(E \simeq 20 \mathrm{MeV})$. This may imply that the nonperturbative part of the one-pion exchange contribution in two-pion exchange diagrams is not properly renormalized in the higher momentum region, $p>137 \mathrm{MeV}$, and the perturbative treatment in the KSW scheme for the ${ }^{3} S_{1}$ channel, even including the di-baryon field, is stalled [12]. This issue is indeed beyond the scope of the present our work including up to the one-pion-exchange contribution, and we may come back to it in the future.

Though the scale parameter $\mu$ has been conventionally fixed as $\mu=m_{\pi}$, we present that the variation of $\mu$ provides us a simple and useful test to investigate the range of the applicability of the theory. What we have obtained above may just imply a confirmation 
of a previous result; the slow convergence of the corrections from the perturbative pions, but we have newly and quantitatively investigated the range of applicability of the theory as a function of the scale parameter $\mu$.

\section{Acknowledgements}

We thank Tae-Sun Park for useful comment to our work and discussions. This work was supported by the Daegu University Research Grant, 2011.

\section{References}

[1] M. C. Birse, J. A. McGovern, and K. G. Richardson, Phys. Lett. B 464 (1999) 169.

[2] S. Scherer, Adv. Nucl. Phys. 27 (2003) 277.

[3] V. Bernard, Prog. Part. Nucl. Phys. 60 (2008) 82.

[4] H. A. Bethe, Phys. Rev. 76 (1949) 38.

[5] H. A. Bethe and C. Longmire, Phys. Rev. 77 (1950) 647.

[6] S. Weinberg, Phys. Lett. B 251 (1990) 288.

[7] S. Weinberg, Nucl. Phys. B 363 (1991) 3.

[8] P. F. Bedaque and U. van Kolck, Annu. Rev. Nucl. Part. Sci. 52 (2002) 339.

[9] E. Epelbaum, H.-W. Hammer, Ulf-G. Meißner, Rev. Mod. Phys. 81 (2009) 1773.

[10] R. Machleidt and D.R. Entem, Phys. Rep. 503 (2011) 1.

[11] U. van Kolck, Nucl. Phys. A 645 (1999) 273.

[12] S. R. Beane, P. F. Bedaque, M. J. Savage, and U. van Kolck, Nucl. Phys. A 700 (2002) 377.

[13] D. B. Kaplan, M. J. Savage, and M. B. Wise, Phys. Lett. B 424 (1998) 390.

[14] D. B. Kaplan, M. J. Savage, and M. B. Wise, Nucl. Phys. B 534 (1998) 329.

[15] D. B. Kaplan, Nucl. Phys. B 494 (1997) 471.

[16] S. R. Beane and M. J. Savage, Nucl. Phys. A 694 (2001) 511.

[17] S. Ando and C. H. Hyun, Phys. Rev. C 72 (2005) 014008.

[18] J. Soto and J. Tarrús, Phys. Rev. C 78 (2008) 024003.

[19] E. Braaten and H.-W. Hammer, Phys. Rev. Lett. 91 (2003) 102002. 
[20] E. Epelbaum, H.-W. Hammer, U.-G. Meißner, and A. Nogga, Eur. Phys. J. C 48 (2006) 169.

[21] T. Barford and M. C. Birse, Phys. Rev. C 67 (2003) 064006.

[22] S. Fleming, T. Mehen, I.W. Stewart, Nucl. Phys. A 677 (2000) 313.

[23] S.R. Beane, D.B. Kaplan, A. Vuorinen, Phys. Rev. C 80 (2009) 011001.

[24] T. D. Cohen and J. M. Hansen, Phys. Rev. C 59 (1999) 13.

[25] T. D. Cohen and J. M. Hansen, Phys. Rev. C 59 (1999) 3047.

[26] B. Dimm, FeynDiagram Version 2.6, http://feyndiagram.com.

[27] H. W. Grießhammer, Nucl. Phys. A 744 (2004) 192.

[28] J. Soto and J. Tarrús, Phys. Rev. C 81 (2010) 014005.

[29] B. Long and C.-J. Yang, Phys. Rev. C 84 (2011) 057001.

[30] NN-OnLine, http://nn-online.org.

[31] C. H. Hyun, T.-S. Park and D.-P. Min, Phys. Lett. B 473 (2000) 6.

[32] E. Epelbaum and J. Gegelia, Eur. Phys. J. A 41 (2009) 341.

[33] T. Mehen and I. W. Stewart, Phys. Lett. B 445 (1999) 378. 